\title{
Virtual Sensors of Tire Pressure and Road Friction
}

Fredrik Gustafsson, Niclas Persson

Markus Drevo, Mats Lofgren, Henrik Quicklund, Urban Forssell

Division of Communication Systems

Department of Electrical Engineering

Linköpings universitet, SE-581 83 Linköping, Sweden

WWW: http://Www.comsys.isy.liu.se

Email: fredrik@isy.liu.se, persson@isy.liu.se

27th August 2001

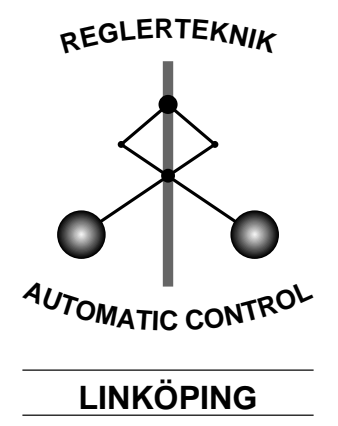

Report No.: LiTH-ISY-R-2376

Submitted to SAE'01, Detroit, MI, USA

Technical reports from the Communication Systems group in Linköping are available by anonymous ftp at the address ftp.control.isy.liu.se. This report is contained in the file 2376 pdf. 


\begin{abstract}
The idea of a virtual sensor is to extract information of parameters that cannot be measured directly, or at least would require very costly sensors, by only using available information. Virtual sensors are described for the friction between road and tire, the tire inflation pressure and wheel imbalance. There are certain in-terconnections between these virtual sensors so they are preferably implemented in one unit. Results from a real-time implementation, using mainly sensor in-formation from the CAN bus, are given
\end{abstract}

Keywords: Sensor, Fusion, Friction, Tire, Pressure, Virtual 


\author{
Fredrik Gustafsson \\ Department of Electrical Engineering, Linköping University, Sweden \\ Markus Drevö, Urban Forssell, Mats Löfgren, Niclas Persson, Henrik Quicklund
}

Copyright (c) 2001 Society of Automotive Engineers, Inc.

NIRA Dynamics AB

\begin{abstract}
The idea of a virtual sensor is to extract information of parameters that cannot be measured directly, or at least would require very costly sensors, by only using available information. Virtual sensors are described for the friction between road and tire, the tire inflation pressure and wheel imbalance. There are certain interconnections between these virtual sensors so they are preferably implemented in one unit. Results from a real-time implementation, using mainly sensor information from the CAN bus, are given.
\end{abstract}

\section{INTRODUCTION}

By a virtual sensor we mean an algorithm that computes a value of a variable which is not measured directly. The reason might be that the variable is not possible to measure, or that a dedicated sensor would be very costly. Instead, statistical inference is made from a model that explains how other (existing) sensor signals depend on the unmeasured variable.

The problems of road tire friction, tire pressure and wheel imbalance might seem far apart. However, the virtual sensors described here use exactly the same sensor signal, namely the wheel speed sensors. These can be taken from the CAN bus, except for the vibration analysis to be described. The tools are also partly the same: a Kalman filter estimates the parameters in a simple model, where the model is not intended to describe very complicated physical phenomena, but rather an instrument to describe what can be observed. Another coupling is that one of the parameters in the friction model, contains very useful information about the tire pressure, so the outline is that friction estimation is described first, then different approaches to tire pressure estimation are compared. Tire imbalance detection is a direct spinoff of a certain approach to tire pressure indication, and is only briefly commented upon there.

NIRA Dynamics AB is together with Linköping University, developing adaptive filters for automotive applications. As one project, real-time hardware has been developed containing algorithms for friction estimation and tire pressure estimation. The aim of this contribution is to present the results and ideas of these algorithms.

\section{FRICTION ESTIMATION}

The road friction indication (RFI) project is a direct application of previous work reported in Gustafsson (1997) and Gustafsson (1998). As briefly outlined in Figure 1, the longitudinal wheel slip and the wheel torque are computed from standard sensor signals. Then a Kalman filter estimates a linear relation between these, and the slope of the line is mapped to a surface in a classifier.

The mathematical model is now briefly outlined. The classical tire model as described in for instance Gillespie (1992), Adler (1993) and Wong (1993), defines a slip curve as the one shown in Figure 1. The wheel slip is usually defined as the relative speed of 


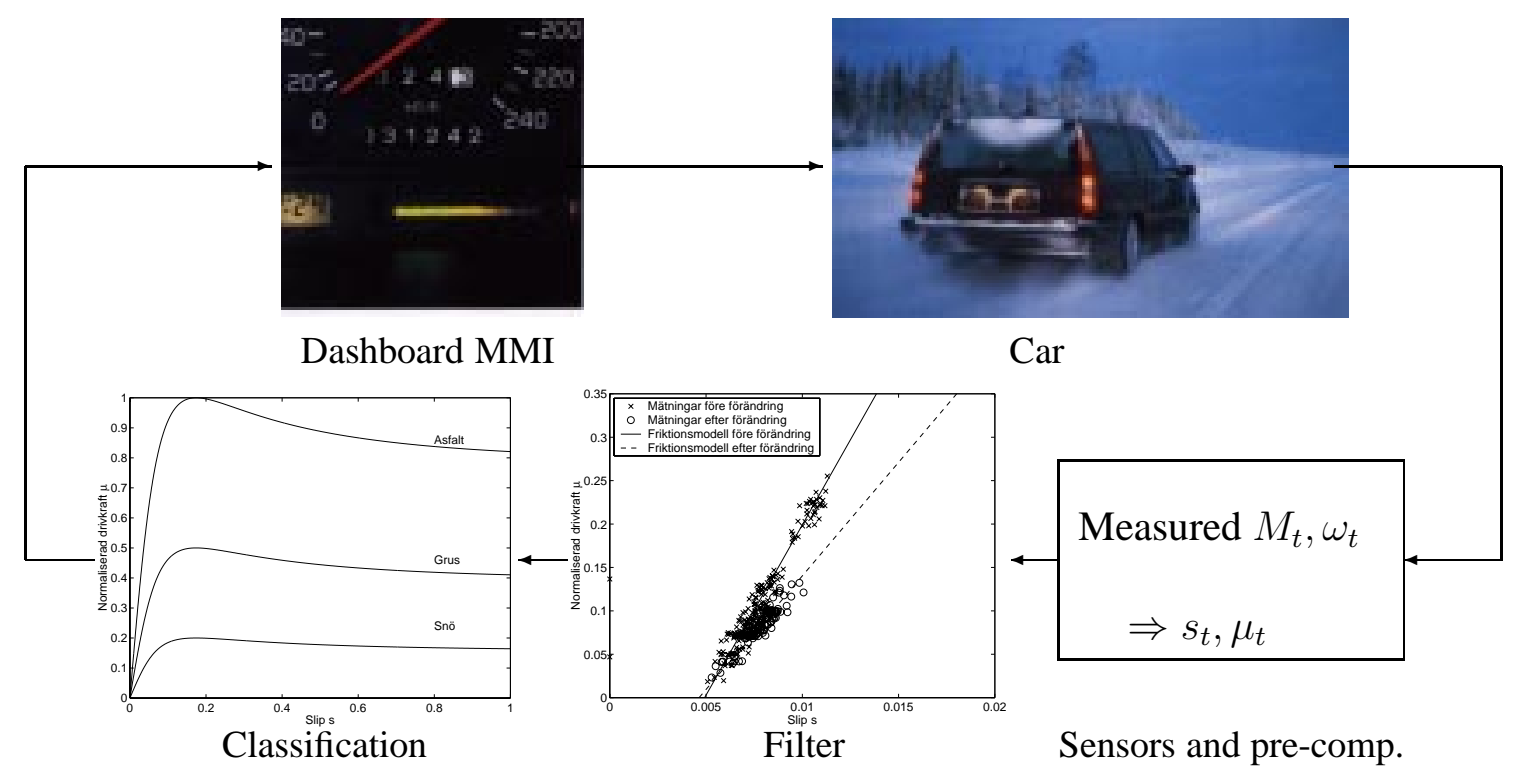

Figure 1: Overview of the RFI algorithm. 1. Sensors: The individual wheel speeds $\omega_{t}^{i}$ and engine torque indicator $M_{t}$ (injection time or manifold pressure) are taken from the CAN bus. 2. Pre-computations: From these, normalized traction force $\mu_{t}$ and wheel slip $s_{t}$ are computed. 3. Filter: A Kalman filter adapts a linear relationship bewteen these (the plot shows measurements from two different surfaces and an estimated straight line). 4. Classification: The slope of the linear relationship is matched to a slip curve (the plot shows three examples on different friction levels). 5. MMI: The driver is alerted for low friction by a warning signal.

a tire compared to its circumferential speed:

$$
s=\frac{\omega r}{v_{x}}-1 .
$$

For small values of the normalized traction force $\mu=$ $F_{x} / F_{z}$ (sometimes called friction utilization coefficient), the wheel slip is a linear function $\mu=k s$ which depends only on tire characteristics. The new finding is that it also depends on friction. A state space model for the left side of the vehicle uses the state vector $x_{t}=\left(1 / k_{l}, \delta_{13}\right)=$ (inverse longitudinal stiffness, relative difference in tire radius between front and rear wheel):

$$
\begin{aligned}
x_{t+1} & =x_{t}+v_{t} \\
s_{t} & =\left(\mu_{l, t}, 1\right) x_{t}+e_{t} .
\end{aligned}
$$

An independent and analogous model is used on the right side of the vehicle. That is, the model says that the measured wheel slip is a linear function of the normalized traction force $\mu$ plus an offset and additive noise. The offset is mainly caused by unequal tire radius on front and rear wheels. The Kalman filter applied directly on the state space model, and a careful tuning gives a decent trade-off between noise attenuation and tracking speed after abrupt changes. It is here useful to give the two parameters in the state vector unequal adaptation gain, such that friction changes can be tracked faster than a change in $\delta_{13}$. Since it is of utmost importance to distinguish very small differences in slip slope $k$, the filter has to be rather slow. For that reason, a change detection mechanism is needed to speed up the response after a detected friction change. The change detector is also coupled to the driver information unit. The theory of combining Kalman filtering and change detection, with the particular case study of RFI, is described in detail in Gustafsson (2000).

Figure 2 shows a test drive with the current prototype. The estimated slip slope is significantly smaller on the skid pad, and the Kalman filter with change detector react quickly. The response time is about 2 seconds, which would be enough for driver alert and control systems as adaptive cruise control.

Improvements in the current project, over previously reported work, include:

- A calibration procedure (what is the normal slope on asphalt for the used tires?) This was in Gustafsson (1997) and Gustafsson (1998) rec- 


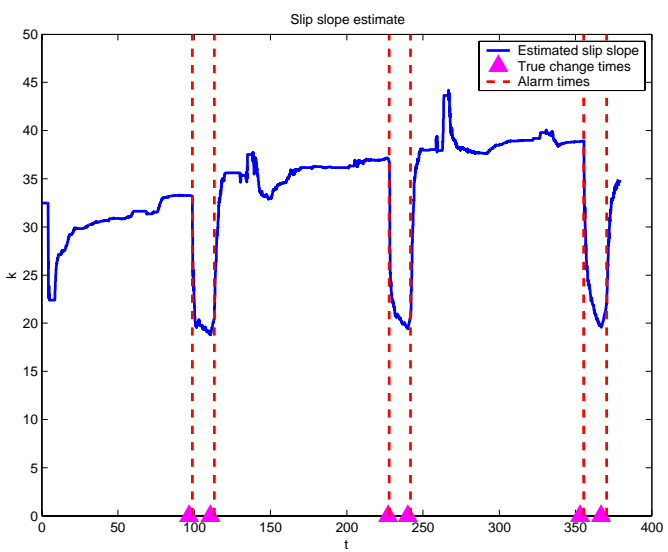

Figure 2: Estimated slip slope while repeatedly driving on and off a skid pad. Mean delay for detection is 2.2 seconds.

ognized as the main problem to be overcome.

- Another Kalman filter using the lateral slip (the lateral forces are for many drivers larger than the longitudinal ones).

- Slip computation using absolute velocity estimation (reported in an accompanying paper) enabling $4 W D$ vehicles to have RFI.

- Aqua planing detection interpreting excessive slip in the friction model as aqua planing. Compared to a direct apprach based on thresholding the slip value directly, we are here able to have a much more sensitive system which compensates for the offset and dynamic effects during acceleration.

We will here, however, focus on RFI as one tool for designing a good tire pressure estimation system. It turns out that the offset $\delta_{13}$ has certain nice properties for this application.

\section{INTRODUCTION TO TIRE PRESSURE IN- DICATION}

There are plenty of on-going $R \& D$ projects on tire pressure indication (TPI), reflected in more than 40 patents. The most relevant for this study are EP700798 (1996), Takeyasu (1997), Yoshihiro (1998), Hideki (1999), Toshiharu (1999) and Fritz (2000). There are two classes of patents using standard sensors (wheel speeds):

- Vibration analysis using the fact that the rubber in the tire reacts like a spring when excited by road roughness. Characteristic features are a high sampling frequency on wheel speed, and disturbance rejection from vibration caused by other moving parts in the vehicle. The vibration analysis can be performed by FFT-based methods or by parametric methods (using an auto-regressive model).

- Wheel radius estimation and comparison. The most common suggestion is to monitor a residual based on a static non-linear transformation of wheel speeds which should be close to zero when the tires are equally large. This residual is then averaged and compared to a decision threshold.

In both approaches a calibration button is often suggested, which must be pressed when one or more tires are changed, or when the pressure is adjusted. When pressed, the algorithm computes what the nominal value of the resonance frequency or residual is.

This paper describes the result of two improvements suggested in Persson and Drevö (1999) and Gustafsson et al. (2000) and now tested in practice:

- Improving the vibration analysis with refined disturbance rejection and signal processing for estimation of auto-regressive parameters. The gain of the patent pending idea is to run the filter at a low sampling frequency $(20 \mathrm{~Hz})$, and achieve twice as accurate result using a Kalman filter.

- As suggested in Gustafsson (1997) and investigated in Jonsson (1993), the so called slip offset from the RFI Kalman filter is a good indicator of relative wheel radius. It is a linear function of data, and is thus not as sensitive to noise as other suggestions. The slip offset estimates the relative difference between left and right wheel pairs. As a complement and further support, the relative difference between front and rear wheel pairs is computed by the yaw rate filter described in an accompanying paper, 
which further has the potential to compute absolute wheel radius.

\section{VIBRATION ANALYSIS FOR TIRE PRES- SURE INDICATION}

The basic idea is that the tire can be seen as a number of connected spring-damper systems. For instance the tire rim is one and the tire pattern another. The most distinguished vibration mode is between 40 and 50 Hertz, depending on the tire model and pressure, but there are other modes of both higher and lower frequencies. The idea is to monitor the vibration frequency, to decide what the normal value is, either by learning or voting between the different tires, and to detect abnormal values.

\section{Calibration of toothed wheel}

Before vibration analysis is applied, no matter if its model-based or FFT-based, the speed measurements have to be filtered. The reason is that the toothed wheel, see Figure 3, is not perfect, and the angular errors from manufacturing and aging, will show up in the frequency content of the speed signal.

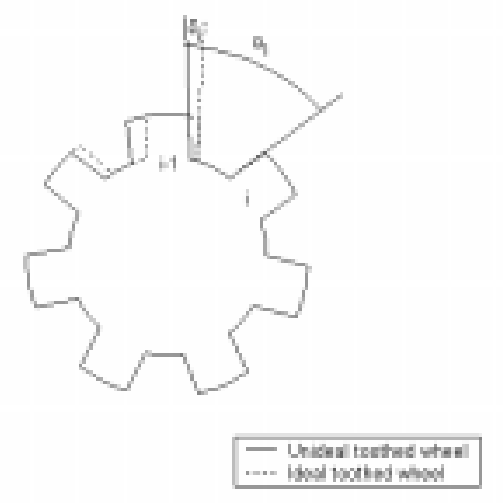

Figure 3: Schematic picture of a toothed wheel with non-equal tooth sizes.

Figure 4 shows that the interesting information is hardly visible because of the disturbance from the toothed wheel. Here the disturbance looks narrowbanded and well-behaved, but in a drive where ve- locity is changed the energy leaks out to other frequencies.

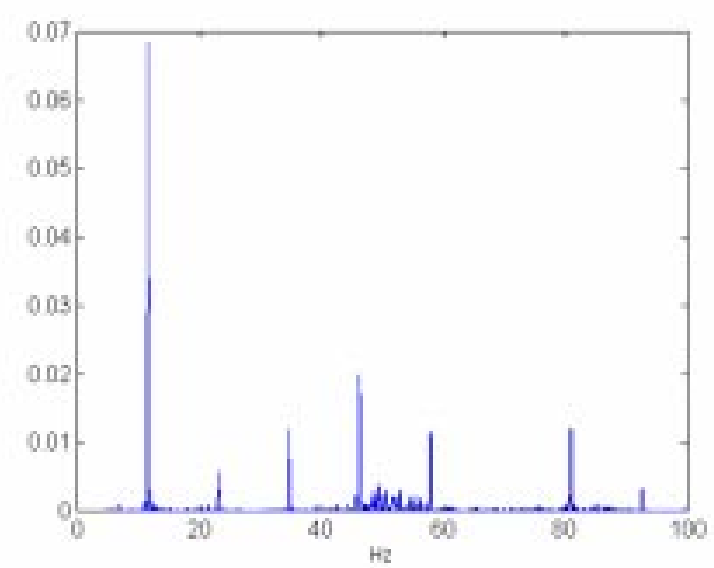

Figure 4: Spectrum of un-processed wheel speed data, where the peaks come from imperfections in the toothed wheel. When velocity is varied, the peaks are blurred out by leakage effects, and the information in the interesting frequency interval is damaged.

A model-based approach to estimate the offsets $\delta_{i}$ in Figure 3 has been implemented, where the recursive least squares algorithm is used. The result is an explicit estimate of each individual tooth, see Figure 6 , and a speed signal with much improved signal to noise ratio. The spectrum of the compensated speed signal clearly shows a vibration mode around $45 \mathrm{~Hz}$, see Figure 5.

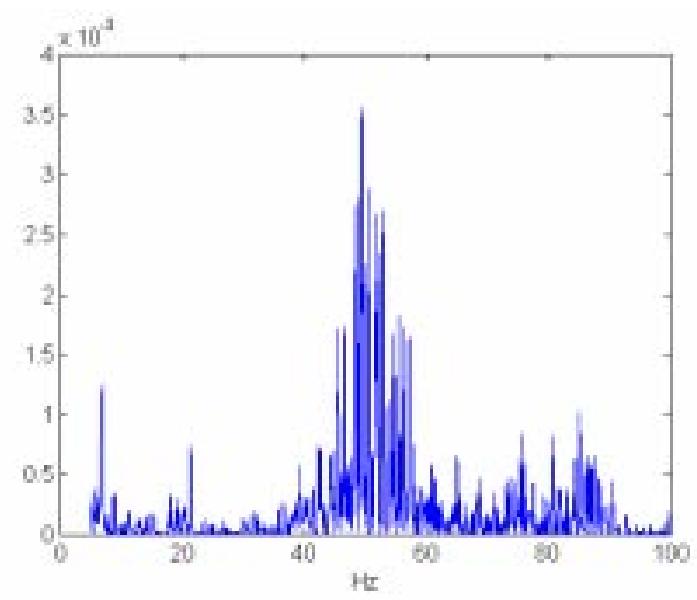

Figure 5: Spectrum of processed wheel speed data. Now the resonance frequency around 45 Hertz is clear. Other resonances can also be distinguished. 
The tooth offsets in Figure 6 can be used for tire imbalance detection. An imbalance will show up as a certain pattern in the estimated sequence. Experiments have shown that an imbalance caused by a 30 gram weight is detectable by this method.

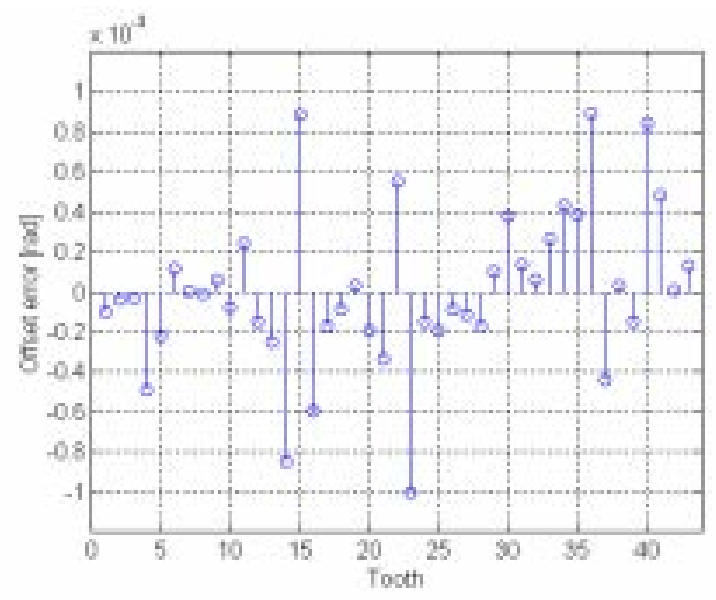

Figure 6: The estimated teeth offsets from a toothed wheel with 43 teeth.

This pre-processing means that the equipment has to be connected directly to the wheel speed sensors. That is, one drawback with vibration analysis not shared by the approach in Section 5, is that CAN signals are not enough.

\section{Evaluation setup}

To evaluate the advantages and short-coming of the different approaches, a series of test drives were conducted:

- The pressure was decreased by $15 \%$ and $30 \%$, respectively. For each pressure level, the driving style, surface and velocity were varied systematically.

- The methods were compared with respect to their ability to detect small pressure changes, robustness to the factors above, and detection time.

\section{FFT pressure estimation}

A smoothed periodogram is here computed using FFT and a low-pass filter. The automatically computed peak level for each test drive is shown in Figure 7. Off-line, a pressure decrease of $15 \%$ is detectable, but taking into account that the treshold has to be set automatically and that a very low false alarm rate is required, $30 \%$ decrease is more realistic. FFT is a batch-wise data processing, so there is a certain delay. In the figure, batches of 30 seconds of drive is used. A drawback with the approach is a rather high computational burden.

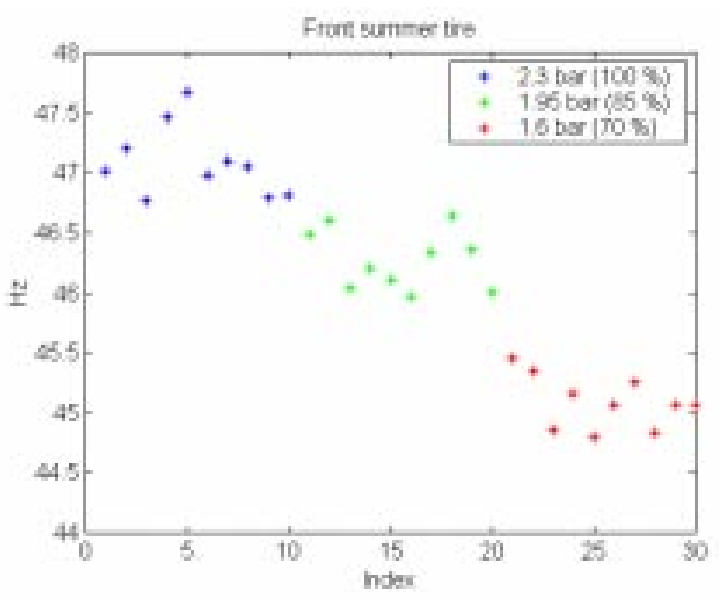

Figure 7: Vibration mode for different tire pressures for 30 tests.

\section{Model-based pressure estimation}

The idea is to estimate the parameters in a second order damper-spring model of the form

$$
v_{t}=\frac{1}{1+a_{1} q^{-1}+a_{2} q^{-2}} e_{t},
$$

where $v_{t}$ is the wheel speed, $e_{t}$ an unmeasured noise signal and $q$ is the shift operator $q^{-1} v_{t}=v_{t-1}$. The parameters $a_{1}, a_{2}$ correspond to a certain vibration mode. Model-based vibration analysis easily allows a recursive implementation, so the vibration mode as a function of time using the recursive least squares (RLS) algorithm is shown in Figure 8. With this filter, we can get reliable detection of $30 \%$ pressure decrease within 5 seconds. The adaptive filter is processing data at least with $100 \mathrm{~Hz}$. The computation burden is slighly smaller but comparable with the FFT-based approach.

The novel approach uses a Kalman filter which is run in only $20 \mathrm{~Hz}$. This filter has more degrees of 


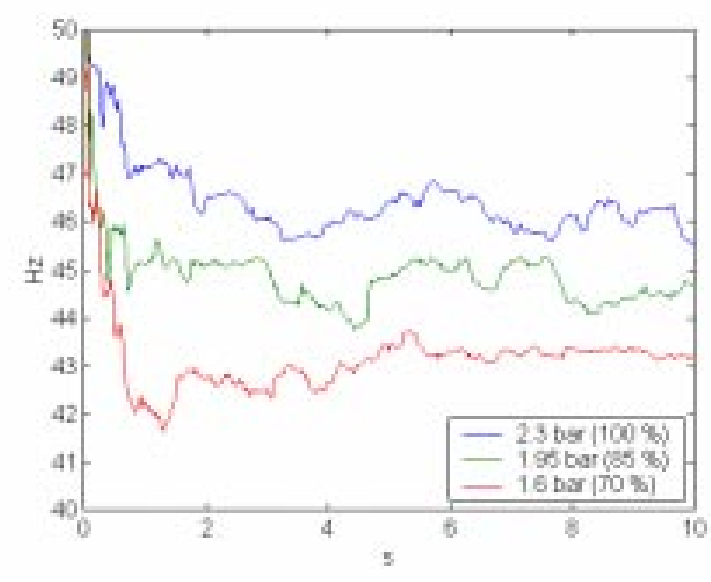

Figure 8: Estimated vibration mode using RLS as a function of time for three pressure levels.

freedom at the same computational burden as RLS, allowing a much better design for this purpose. Holding the tracking speed of RLS and Kalman filter the same, Figure 9 shows a much more stable estimate. That is, the new implementation is able to detect $15 \%$ pressure changes as fast as RLS detects $30 \%$ changes, with an implementation that requires almost 5 times less computations.

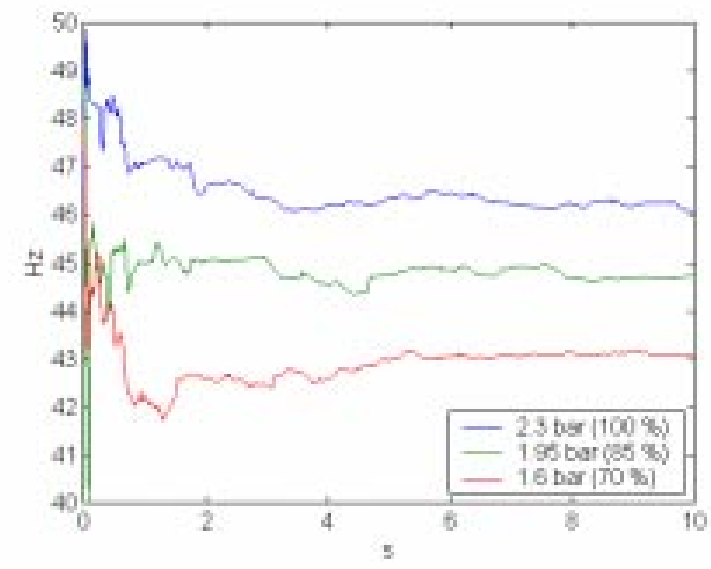

Figure 9: Estimated vibration mode using the Kalman filter as a function of time for three pressure levels.

\section{WHEEL RADIUS APPROACH TO TIRE PRES- SURE INDICATION}

Existing approaches to TPI are mostly based on static consistency tests on wheel speeds, where the test statistic is possibly low-pass filtered. As one example, not pursued here, one can monitor

$$
\frac{\omega_{1}}{\omega_{2}}-\frac{\omega_{3}}{\omega_{4}}
$$

The wheel speeds $\omega_{i}$ are enumerated as front left (1), front right (2), rear left (3) and rear right (4) wheel, respectively. This function of wheel speed is nominally zero when driving with constant speed on straight paths or circle segments. A low-pass filtered version of this signal having an abnormal deviation from 0 exceeding a certain threshold can be used for TPI. However, the non-linear transformation of data gives unpleasant statistical behavior, and the lack of robustness to acceleration in bends, different friction levels, "split $\mu$ ", tire wearness and so on limit the reliability of this approach.

We have found that a model-based approach which explicitly takes care of all the problems listed above gives a significant increase in performance. The modelbased filters that are used here, are the road friction indicator (RFI) filter described in Section 2, high performance yaw rate (HPY) and absolute velocity indicator (AVI) filters, the latter two ones described in an accompanying paper, are instrumental for TPI. The useful information is summarized in Table 1.

The deviation $\delta_{r}$ of average radius to nominal value may be used for detecting diffusion, that is when all tires deflate slowly.

The conclusion from field tests were all approaches have been compared, is that this latter method is superior in performance in terms of accuracy, fast response, robustness and computational complexity (loworder models and low sampling frequency).

However, the other approaches may be a good complement in certain situation, and the final system may be a voting procedure of the individual algorithms. For instance, the absolute difference $\delta_{r}$ is much harder to estimate than relative differences $\delta_{i j}$, so diffusion is a possible problem here, where vibration analysis may perform better. 


\begin{tabular}{|c|l|}
\hline Radius difference & Estimation approach \\
\hline$\delta_{13}$ & RFI, left side of car \\
$\delta_{24}$ & RFI, right side of car \\
$\delta_{12}$ & HPY, front axle \\
$\delta_{34}$ & HPY, rear axle \\
$\delta_{r}$ & AVI \\
\hline
\end{tabular}

Table 1 Definition and estimation of relative tire radius differences. The wheels are enumerated as front left (1), front right (2), rear left (3) and rear right (4) wheel, respectively. For example, $\delta_{12}$ is defined as $\left(r_{1}-\right.$ $\left.r_{2}\right) / r_{\text {nom }}$, where $r_{\text {nom }}$ is the nominal radius, and $r_{i}$ the tire radius on wheel $i . \delta_{r}$ denotes the actual mean tire radius minus the nominal value.

\section{CONCLUSIONS}

Intelligent sensors measure information indirectly, by making inference from other sensor signals. Two examples are road-tire friction and tire pressure, which are very expensive to measure by dedicated sensors. By measuring wheel speed signals accurately, it has been demonstrated that inference of both friction and pressure can be made. The novelty of friction estimation here is a working prototype now approaching the production phase. For tire pressure estimation, two suggestions for improving performance were made. First, for vibration analysis a model based Kalman filter was proposed, which gives higher estimation accuracy compared to algorithms proposed in literature, and an update frequency being only a fraction of existing methods. Second, a wheel radius modelbased method is advocated. This is robust to a variety of external factors, quick reaction time, computationally simple and needs only information available on the CAN bus.

\section{REFERENCES}

Adler, Dipl.-Ing.(FH) Ulrich (1993). Automotive Handbook, 3rd ed. Robert Bosch GmbH.

EP700798 (1996). Tire pneumatic pressure detector. EP700798, US 5606122. Nippon Denso Co, Nip- pon Soken.

Fritz, Braun (2000). Method and device for monitoring the tire air pressure of the wheels of an automobile. EP938987, US 6092415, DE19807880. Daimler Chrysler AG.

Gillespie, T.D. (1992). Fundamentals of Vehicle Dynamics. SAE International.

Gustafsson, F. (1997). Slip-based estimation of tire road friction. Automatica, 33(6), 1087-1099.

Gustafsson, F. (1998). Estimation and change detection of tire - road friction using the wheel slip. IEEE Control System Magazine, 18(4), 42-49.

Gustafsson, Fredrik (2000). Adaptive filtering and change detection. John Wiley \& Sons, Ltd.

Gustafsson, F., M. Drevö, and N. Persson (2000). Tire pressure computation system. Swedish patent application nr 0002213-7.

Hideki, Kabune (1999). Apparatus for estimationg tire air pressure. EP865880, US 6092028. Denso Corp.

Jonsson, H. (1993). Examination and tools for classification of tire road friction. Master Thesis LiTHISY-EX-1396, Dept of Elec. Eng. Linköping University, S-581 83 Linköping, Sweden. In Swedish.

Persson, N. and M. Drevö (1999). Tire pressure estimation for cars. Master Thesis LiTH-ISY-EX3033, Dept of Elec. Eng. Linköping University, S58183 Linköping, Sweden.

Takeyasu, Taguchi (1997). Tire air pressure detecting device. EP783982. Nippon Denso Co.

Toshiharu, Naito (1999). Tyre air pressure estimating apparatus. EP925960. Denso Corp.

Wong, J.Y. (1993). Theory of ground vehicles, 2nd ed. John Wiley \& Sons, Inc.

Yoshihiro, Nishikawa (1998). Tire air pressure detecting device. EP832768, US 5982279. Denso Corp, Nippon Soken. 